\title{
Neurointensive Care Unit and neurointensivist: Do we need them?
}

Neurocritical/neurointensive care is a branch of medicine that came into existence in the 1980s. It deals with the life-threatening diseases of the brain, spinal cord and nerves. The development of neurointensive care subspeciality was largely prompted by high-incidence of medical errors and insufficient care in the US. In response, physicians in medicine, anaesthesia and critical care began working together to provide highly specialised care in collaborative setting. ${ }^{[1]}$ Neurointensivists are specialists with skills to manage various aspects of these critically ill neurology/neurosurgical patients in Neurointensive Care Unit (NICU). Neurocritical care is the mainstay of an all important link between brain and other body systems in these patients. NICU is an ideal place to address the unique requirements of these critically ill patients. For satisfactory management of these patients, it has become imperative to carve out a separate NICU instead of treating them in a general Intensive Care Unit (ICU). While neurointensive care has already established itself as a subspeciality in the Western world, in developing countries including India, it is still fledgling. The scope of neurointensive care has been increasing by leaps and bound with the advent of new diagnostic techniques, new therapies and ageing population. The NICU provides a unique place where a neurointensivist can better appreciate the nuances of neurologic disease process.

In addition to standard interventions, a neurocritical care expert offers specific care including monitoring of intracranial pressure, cerebral haemodynamic improvement, therapeutic hypothermia and advanced monitoring (brain oximetry, cerebral microdialysis and continuous electroencephalography). As understanding of secondary brain injury following traumatic brain injury (TBI) has advanced, increased attention is being paid to ICU management of these patients, specifically with regard to prevention, identification and treatment of secondary brain insult. Because of increased complexity of neuro diseases, a multidisciplinary approach to neurocritical care is advocated. Neurointensivists in collaboration with neurosurgeons, neurologists and other physicians implement the acute care protocols decisions in NICU. Neurocritical care field is open at present to neurologists, neurosurgeons, anaesthesiologists and critical care specialists.

NICU is also a place for the effective management of various critically ill neurological patients as well serious post-operative neurosurgical patients. Outcome research has established that as a result of care by a dedicated neurointensivist in NICU charged with managing all aspects of these patients (as opposed to care provided in general ICU), victims of TBI, haemorrhagic stroke and subarachnoid haemorrhage have lower mortality, better functional outcome and reduced length of stay. The team-based approach using neurointensivist to manage neurosurgical subarachnoid haemorrhage patients, merits further study of a successful model of care. ${ }^{[2]}$ This improved outcome may not benefit patients of intracranial haematoma who tend to have poor outcomes regardless of the presence of a neurocritical care unit and neurointensivist. ${ }^{[3]}$ Recent data have highlighted favourable outcome and lower mortality in neurosurgical patients attended to by a team of devoted neurointensivists. ${ }^{[4,5]}$ It is now known that neurological/neurosurgical patients undergo more invasive intracranial and haemodynamic monitoring, tracheostomy and nutritional support and receive less intravenous sedation than patients in general ICU. ${ }^{[6]}$ In addition, management of these patients in NICU results in lower cost of care than a National Benchmark of USA. ${ }^{[7]}$

Varelas et al. ${ }^{[8]}$ compared outcomes of patients treated before and after implementation of neurointensivists in the ICU and identified three critical factors that improved neurosurgical patients' care; (1) implementing a dedicated neurointensivist; (2) attention to details; (3) better monitoring procedures.

On the contrary, according to Suarez et al., in spite of these benefits, change in readmission rates or long-term mortality does not change. ${ }^{[9]}$ This has caused some aspersions on the necessity of NICU. Nonetheless, in a survey Markandaya et al. ${ }^{[10]}$ observed that over $70 \%$ of respondents reported the availability of neurocritical care units staffed with neurointensivists would improve the quality of care of critically ill neurological patients. This survey threw up a surprising observation that neurologists were reported as the most appropriate speciality for training in neurointensive care by $53.3 \%$, and $57 \%$ of the respondents reported positively that neurology residency programme should offer a separate training track for those interested in critical care. However, neurologists and neurosurgeons lack the desired temperament of a neuroanaesthesiologist/anaesthesiologist which is one of the essential prerequisites for the proper management of critically ill neurological/neurosurgical patients. 
Since these patients may be on multisystem monitoring with the need for invasive lines insertion, this gives anaesthesiologists an edge over the other specialists. Moreover, neurology usually attracts trainees whose main focus may not necessarily be the management of complex critically ill patients though of late, introduction of effective therapies for the treatment of acute ischaemic stroke have brought a paradigm shift in their attitude, and they are now increasingly assuming a more aggressive role. ${ }^{[11]}$ Despite change in their attitude, they might still prefer to limit their involvement with the uncomplicated neurologic diseases, outside the ICU.

Therefore, from the many studies, it can be safely assumed that institution of the neurointensivist team in an NICU has a favourable influence on the outcome of neurologically ill patients. All those centres with large neurological/neurosurgical set up should strive to establish NICU attended to by a team of neurointensivists.

\section{Parmod K. Bithal}

Department of Neuroanaesthesiaology and Critical Care, AlIMS, New Delhi, India Address for correspondence: Prof. Parmod K. Bithal, Department of Neuroanaesthesiaology and Critical Care, AIIMS, New Delhi - 110 029, India. E-mail: bithal.parmod@gmail.com

\section{REFERENCES}

1. Gasperino J. The Leapfrog initiative for Intensive Care Unit physician staffing and its impact on Intensive Care Unit performance: A narrative review. Health Policy 2011;102:223-8.

2. Josephson SA, Douglas VC, Lawton MT, English JD, Smith WS, Ko NU. Improvement in Intensive Care Unit outcomes in patients with subarachnoid hemorrhage after initiation of neurointensivist co-management. J Neurosurg 2010;112:626-30.

3. Knopf L, Staff I, Gomes J, McCullough L. Impact of a neurointensivist on outcomes in critically ill stroke patients. Neurocrit Care 2012;16:63-71.
4. Bleck TP. The impact of specialized neurocritical care. J Neurosurg 2006;104:709-10.

5. Kramer AH, Zygun DA. Do Neurocritical Care Units save lives? Measuring the impact of specialized ICUs. Neurocrit Care 2011;14:329-33.

6. Kurtz P, Fitts V, Sumer Z, Jalon H, Cooke J, Kvetan V, et al. How does care differ for neurological patients admitted to a neurocritical care unit versus a general ICU? Neurocrit Care 2011;15:477-80.

7. Mirski MA, Chang CW, Cowan R. Impact of a Neuroscience Intensive Care Unit on neurosurgical patient outcomes and cost of care: Evidence-based support for an intensivist-directed specialty ICU model of care. J Neurosurg Anesthesiol 2001;13:83-92.

8. Varelas PN, Eastwood D, Yun HJ, Spanaki MV, Hacein Bey L, Kessaris C, et al. Impact of a neurointensivist on outcomes in patients with head trauma treated in a Neurosciences Intensive Care Unit. J Neurosurg 2006;104:713-9.

9. Suarez JI, Zaidat OO, Suri MF, Feen ES, Lynch G, Hickman J, et al. Length of stay and mortality in neurocritically ill patients: Impact of a specialized neurocritical care team. Crit Care Med 2004;32:2311-7.

10. Markandaya $M$, Thomas KP, Jahromi B, Koenig $M$, Lockwood AH, Nyquist PA, et al. The role of neurocritical care: A brief report on the survey results of neurosciences and critical care specialists. Neurocrit Care 2012;16:72-81.

11. Da Silva IR, Gomes JA. Residency training: The role of neurocritical care in resident education. Neurology 2013;80:e51-3.

This is an open access article distributed under the terms of the Creative Commons Attribution-NonCommercial-ShareAlike 3.0 License, which allows others to remix, tweak, and build upon the work non-commercially, as long as the author is credited and the new creations are licensed under the identical terms.

\begin{tabular}{|l|l|}
\hline \multicolumn{2}{|c|}{ Access this article online } \\
\hline Quick Response Code: & Website: \\
\hline & www.jnaccjournal.org \\
\cline { 2 - 2 } & \\
\hline
\end{tabular}

How to cite this article: Bithal PK. Neurointensive Care Unit and neurointensivist: Do we need them?. J Neuroanaesthesiol Crit Care 2016;3:1-2. 